\title{
Low phase angle effects in photometry of trans-neptunian objects: 20000 Varuna and $19308\left(1996 \mathrm{TO}_{66}\right)$
}

\author{
I.N. Belskaya ${ }^{\mathrm{a}, \mathrm{b}, *}$, J.L. Ortiz $^{\mathrm{c}}$, P. Rousselot ${ }^{\mathrm{a}}$, V. Ivanova ${ }^{\mathrm{d}}$, G. Borisov ${ }^{\mathrm{d}}$, \\ V.G. Shevchenko ${ }^{\mathrm{b}}$, N. Peixinho ${ }^{\mathrm{e}}$ \\ a Observatoire de Besançon, 25000 Besançon, France \\ $\mathrm{b}^{\mathrm{b}}$ Astronomical Institute of Kharkiv National University, Sumska str. 35, Kharkiv 61022, Ukraine \\ ${ }^{\mathrm{c}}$ Instituto de Astrofísica de Andalucía, CSIC, Apt 3004, 18080 Granada, Spain \\ d Institute of Astronomy, Bulgarian Academy of Sciences, BG-1784 Sofia, Bulgaria \\ e GAUC, Observatório Astronómico da Universidade de Coimbra, P-3040 Coimbra, Portugal
}

Received 11 February 2006; revised 19 April 2006

Available online 8 June 2006

\begin{abstract}
We present the results of photometric observations of trans-neptunian object 20000 Varuna, which were obtained during 7 nights in November 2004-February 2005. The analysis of new and available photometric observations of Varuna reveals a pronounced opposition surge at phase angles less than $0.1 \mathrm{deg}$ with amplitude of 0.2 mag relatively to the extrapolation of the linear part of magnitude-phase dependence to zero phase angle. The opposition surge of Varuna is markedly different from that of dark asteroids while quite typical for moderate albedo Solar System bodies. We find an indication of variations of the scattering properties over Varuna's surface that could result in an increase of the lightcurve amplitude toward zero phase angle. It is shown that a similar phase effect can be responsible for lightcurve changes found for $\mathrm{TNO}_{19308}\left(1996 \mathrm{TO}_{66}\right)$ in 1997-1999.
\end{abstract}

(C) 2006 Elsevier Inc. All rights reserved.

Keywords: Trans-neptunian objects; Asteroids; Photometry

\section{Introduction}

The discovery of a large number of objects beyond the orbit of Neptune caused a great interest to study their properties. Trans-neptunian objects (TNOs) are believed to be the most pristine and thermally unprocessed bodies in the Solar System containing unique information on the primordial processes that governed the evolution of our planetary system (e.g., Luu and Jewitt, 2002). Their study is of great importance to understand the key questions of their origin and interrelations between different classes of minor bodies. Presently most of the available information on physical properties for the majority of TNOs comes from their broad-band photometric observations (for review see Barucci et al., 2004).

\footnotetext{
* Corresponding author. Fax: +380 (57) 7005349.

E-mail address: irina@ astron.kharkov.ua (I.N. Belskaya).
}

Ground-based observations of trans-neptunian objects are strictly limited by small phase angles (usually less than $2^{\circ}$ ). At these phase angles the opposition effect phenomenon (OE) should play a dominant role. The opposition effect inherent for solid planetary surfaces manifests in a considerable increase of surface brightness as the phase angle decreases to zero. The amplitude and width of the opposition effect depends on the physical characteristics of the surfaces (e.g., Hapke, 2002). The OE amplitude, defined as an excess of linear approximation of a magnitude-phase curve at zero phase angle, can reach $0.2-0.5 \mathrm{mag}$ and its width varies typically from $10^{\circ}$ to less than $1^{\circ}$ (Buratti et al., 1992; Belskaya and Shevchenko, 2000; Schaefer and Tourtellotte, 2001). Such considerable increase in brightness should not be neglected when observations carry out at small phase angles. Thus, the study of TNOs opposition effect is very important to be taken into account in various kinds of data analysis, particularly regarding their size esti- 
mations. On the other hand, TNOs are the ideal candidates to study backscattering phenomena as they represent a unique possibility to reach practically zero phase angle. Detailed measurements of the opposition effect of TNOs down to extremely small phase angles will provide important information about their surface properties and will give an additional basis to search for resemblances and differences between various types of primitive bodies.

The first phase functions' measurements of TNOs have shown nearly linear phase curves with steep phase slopes (Sheppard and Jewitt, 2002; Schaefer and Rabinowitz, 2002; Rousselot et al., 2003). Based on published observations, Belskaya et al. (2003a) concluded that in addition to the steep phase slopes TNOs seemed to have very narrow opposition surges of about $0.1-0.2 \mathrm{mag}$ at phase angles less than $0.1^{\circ}-0.2^{\circ}$. To check this conclusion we carried out detailed photometric observations of one of the brightest TNOs 20000 Varuna, which are described in Section 2. In Section 3, we analyze all available photometric observations of Varuna and discuss an indication of the lightcurve variability at low phase angles. We also check whether low phase angle effects can cause the lightcurve changes observed for TNO 193081996 TO$_{66}$ (Hainaut et al., 2000; Sekiguichi et al., 2002). The obtained results on opposition effect behavior are discussed in Section 4.

\section{Observations and data reduction}

Observations were carried out in November 2004-February 2005 at Sierra Nevada Observatory (Spain) using the $1.5-\mathrm{m}$ telescope during six nights and at the Bulgarian National Astronomical Observatory Rozhen using the 2-m telescope during a single night on December 8 . The observational circumstances are presented in Table 1 including the mid-night time in UT, the geocentric $(\Delta)$ and heliocentric $(r)$ distances, the solar phase angle $(\alpha)$, the observing site, and photometric conditions.

The camera used at the $1.5-\mathrm{m}$ telescope was a high quantum efficiency $2048 \times 2048$ pixel CCD based on Marconi-EEV CCD 42-40 chip. Its technical specifications are given at http:// www.osn.iaa.es. The field of view is $7.8 \times 7.8$ arcmin with 0.23 arcsec per pixel.

Observations were made with the standard Johnson-CronCousins R filter. Each exposure time was typically of $400 \mathrm{~s}$. The object drift rates were less than 0.5 arcsec per the integration time, while the typical seeing was around 1.5 arcsec. For absolute calibration, standard star fields from Landolt (1992) were measured at different airmasses. Details of the observing method are given in Ortiz et al. (2003).

The observations of Varuna at Rozhen Observatory were made with $1024 \times 1024$ pixel CCD camera Photometrics CE200A-SITe (liquid nitrogen cooling) attached to 2-m Ritchey-Chretien-Coude telescope. During these observations binning $2 \times 2$ was used and the resolution was $0.618^{\prime \prime} / \mathrm{px}$. The observations were also carried out in the standard $\mathrm{R}$ filter with an integration time of $300 \mathrm{~s}$. The mean seeing during the night was 1.8 arcsec.

All observations were reduced in the same way using standard IRAF routines, including bias subtraction and flat-field
Table 1

Observational circumstances

\begin{tabular}{lllllll}
\hline Date, UT & & $\Delta(\mathrm{AU})$ & $r(\mathrm{AU})$ & $\alpha(\mathrm{deg})$ & Obs. site & $\begin{array}{l}\text { Com- } \\
\text { ments }^{\mathrm{a}}\end{array}$ \\
\hline 2004 November & 23.2040 & 42.532 & 43.244 & 0.92 & OSN & PHO \\
2004 December & 08.1055 & 42.382 & 43.246 & 0.64 & Rozhen & CLR \\
2005 January & 06.0015 & 42.267 & 43.250 & 0.056 & OSN & CLR \\
2005 January & 07.9982 & 42.269 & 43.250 & 0.083 & OSN & PHO \\
2005 January & 31.9235 & 42.380 & 43.253 & 0.61 & OSN & CLR \\
2005 February & 01.9795 & 42.389 & 43.253 & 0.63 & OSN & PHO \\
2005 February & 10.0992 & 42.466 & 43.254 & 0.79 & OSN & CLR \\
\hline
\end{tabular}

${ }^{a} \mathrm{PHO}$ is photometric and CLR is clear.

correction. DAOPHOT package was applied for the synthetic aperture measurements. Several synthetic apertures were used for the object and field stars. The data presented here were obtained with the aperture that gave the lowest scatter of the data. It corresponded to a radius of 4 to 6 pixels depending on night seeing conditions. For the absolute calibrations the diameter of the aperture was large enough for both Landolt and field stars. Photometric conditions allowed us to obtain absolute calibration for 5 from 7 nights. In the other cases (January 6 and 31) we used the reference stars for which absolute magnitudes were determined from neighboring nights (January 7 and February 1, correspondingly). The observations of Landolt stars were used to compute the zero point of the instrumental magnitudes and the extinction coefficients in the R band. Unfortunately, our observations were carried out in the single band and we were not able to determine the color extinction coefficients. To minimize color extinction effects we used for calibration a set of standard stars with colors similar to those of Varuna. The estimated uncertainties in absolute magnitudes for observations at Sierra Nevada Observatory are not larger than 0.04 mag. Observations made at Rozhen Observatory gave an uncertainty in absolute magnitudes of about $0.1 \mathrm{mag}$. Table 2 contains the results of our photometric observations: MJD time taken for the mean exposure time, apparent $\mathrm{R}$ magnitude and uncertainty at $1-\sigma$ level.

To extract magnitude-phase dependence we need to take into account magnitude variations of Varuna due to rotation. Analysis of our observations has confirmed the rotation period of $6.3436 \mathrm{~h}$ given by Ortiz et al. (2003). The composite lightcurve with this value of rotation period is shown in Fig. 1. The observational data were corrected for light travel time and were normalized to unit distances from the Earth and the Sun. The lightcurves were shifted in magnitude to observations on December 8 and January 31 obtained at the phase angle of $0.6^{\circ}$ and covered the full rotation cycle. For the purpose we used the software developed by Krugly (2003) for reducing individual lightcurves and constructing composite lightcurves based on the Fourier fit criteria. The composite lightcurve of Varuna (Fig. 1) is characterized by a rather symmetrical shape with two pairs of extrema and amplitude of $0.44 \mathrm{mag}$. The difference between two maxima is not larger than $0.03 \mathrm{mag}$. The difference in minima seems to be larger but its value is uncertain due to a scatter of observational data in one of the minima. 
Table 2

Photometric data of 20000 Varuna

\begin{tabular}{|c|c|c|c|c|c|}
\hline MJD & $\mathrm{R}$ & $\sigma \mathrm{R}$ & MJD & $\mathrm{R}$ & $\sigma \mathrm{R}$ \\
\hline 53332.183 & 19.848 & 0.041 & 53377.959 & 19.630 & 0.038 \\
\hline 53332.187 & 19.990 & 0.041 & 53377.965 & 19.535 & 0.034 \\
\hline 53332.202 & 20.028 & 0.041 & 53377.971 & 19.483 & 0.033 \\
\hline 53332.206 & 19.930 & 0.042 & 53377.980 & 19.428 & 0.033 \\
\hline 53332.220 & 19.934 & 0.040 & 53377.986 & 19.374 & 0.032 \\
\hline 53332.225 & 19.916 & 0.041 & 53377.992 & 19.339 & 0.031 \\
\hline 53347.029 & 19.748 & 0.081 & 53377.998 & 19.350 & 0.030 \\
\hline 53347.035 & 19.712 & 0.081 & 53378.004 & 19.322 & 0.032 \\
\hline 53347.039 & 19.710 & 0.081 & 53378.010 & 19.364 & 0.032 \\
\hline 53347.043 & 19.709 & 0.080 & 53378.019 & 19.455 & 0.042 \\
\hline 53347.046 & 19.650 & 0.080 & 53378.025 & 19.500 & 0.032 \\
\hline 53347.050 & 19.589 & 0.080 & 53378.031 & 19.536 & 0.034 \\
\hline 53347.054 & 19.544 & 0.080 & 53378.037 & 19.559 & 0.040 \\
\hline 53347.058 & 19.523 & 0.080 & 53401.856 & 19.967 & 0.044 \\
\hline 53347.061 & 19.491 & 0.079 & 53401.861 & 19.946 & 0.054 \\
\hline 53347.065 & 19.467 & 0.079 & 53401.866 & 19.872 & 0.052 \\
\hline 53347.069 & 19.520 & 0.078 & 53401.871 & 19.895 & 0.059 \\
\hline 53347.073 & 19.414 & 0.078 & 53401.876 & 19.766 & 0.057 \\
\hline 53347.076 & 19.454 & 0.079 & 53401.881 & 19.807 & 0.064 \\
\hline 53347.080 & 19.436 & 0.080 & 53401.886 & 19.817 & 0.049 \\
\hline 53347.084 & 19.506 & 0.080 & 53401.896 & 19.618 & 0.051 \\
\hline 53347.087 & 19.429 & 0.079 & 53401.901 & 19.637 & 0.052 \\
\hline 53347.091 & 19.445 & 0.079 & 53401.906 & 19.590 & 0.054 \\
\hline 53347.095 & 19.510 & 0.078 & 53401.911 & 19.539 & 0.048 \\
\hline 53347.099 & 19.572 & 0.082 & 53401.916 & 19.537 & 0.044 \\
\hline 53347.102 & 19.590 & 0.081 & 53401.921 & 19.535 & 0.051 \\
\hline 53347.106 & 19.572 & 0.080 & 53401.926 & 19.551 & 0.053 \\
\hline 53347.110 & 19.679 & 0.082 & 53401.931 & 19.535 & 0.051 \\
\hline 53347.113 & 19.635 & 0.083 & 53401.936 & 19.602 & 0.046 \\
\hline 53347.117 & 19.743 & 0.084 & 53401.941 & 19.663 & 0.044 \\
\hline 53347.121 & 20.015 & 0.083 & 53401.946 & 19.697 & 0.050 \\
\hline 53347.125 & 19.938 & 0.085 & 53401.951 & 19.758 & 0.054 \\
\hline 53347.128 & 19.929 & 0.080 & 53401.956 & 19.791 & 0.056 \\
\hline 53347.132 & 19.912 & 0.085 & 53401.961 & 19.856 & 0.045 \\
\hline 53347.136 & 20.047 & 0.086 & 53401.966 & 19.889 & 0.052 \\
\hline 53347.139 & 19.805 & 0.083 & 53401.971 & 19.912 & 0.051 \\
\hline 53347.143 & 19.890 & 0.087 & 53401.976 & 19.965 & 0.047 \\
\hline 53347.148 & 19.770 & 0.084 & 53401.981 & 19.962 & 0.048 \\
\hline 53347.152 & 19.738 & 0.086 & 53401.986 & 19.953 & 0.045 \\
\hline 53347.156 & 20.003 & 0.128 & 53401.991 & 19.887 & 0.047 \\
\hline 53347.159 & 19.907 & 0.098 & 53402.970 & 19.485 & 0.040 \\
\hline 53347.163 & 19.762 & 0.082 & 53402.975 & 19.572 & 0.040 \\
\hline 53347.167 & 19.554 & 0.083 & 53402.980 & 19.514 & 0.041 \\
\hline 53347.171 & 19.650 & 0.080 & 53402.984 & 19.493 & 0.040 \\
\hline 53347.174 & 19.546 & 0.081 & 53402.989 & 19.587 & 0.042 \\
\hline 53347.182 & 19.429 & 0.081 & 53411.074 & 19.998 & 0.052 \\
\hline 53375.977 & 19.570 & 0.038 & 53411.079 & 19.739 & 0.051 \\
\hline 53375.983 & 19.548 & 0.035 & 53411.085 & 19.912 & 0.052 \\
\hline 53375.989 & 19.487 & 0.038 & 53411.091 & 20.119 & 0.056 \\
\hline 53375.995 & 19.430 & 0.037 & 53411.097 & 19.965 & 0.050 \\
\hline 53376.001 & 19.334 & 0.040 & 53411.103 & 20.116 & 0.050 \\
\hline 53376.007 & 19.272 & 0.037 & 53411.109 & 20.050 & 0.051 \\
\hline 53376.013 & 19.264 & 0.034 & 53411.115 & 19.794 & 0.051 \\
\hline 53376.019 & 19.202 & 0.039 & 53411.121 & 20.117 & 0.051 \\
\hline 53376.025 & 19.189 & 0.036 & 53411.126 & 19.993 & 0.051 \\
\hline
\end{tabular}

The composite lightcurve was used to normalize observations at different rotation phases to the same surface corresponding to the maximum of the Varuna's lightcurve. The observations on February 10 were not used for phase curve construction because they were made in the minimum of Varuna's rotational cycle with a great scatter. In addition, we did not used

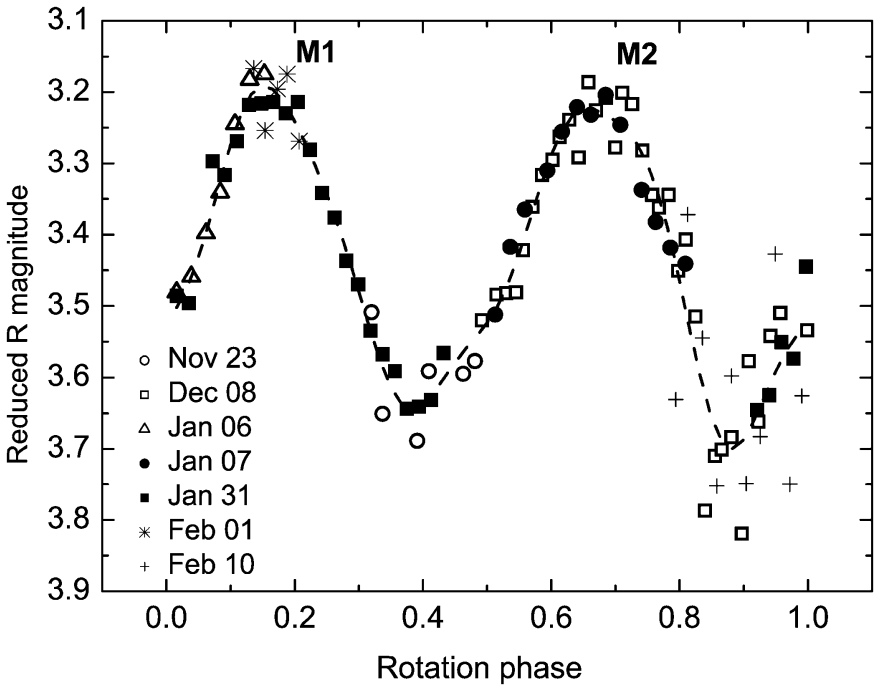

Fig. 1. Composite lightcurve of 20000 Varuna obtained using the rotation period of $6.3436 \mathrm{~h}$ and $\mathrm{JD}_{0}=2453372.0$. The primary and the secondary maxima are marked by $\mathrm{M} 1$ and $\mathrm{M} 2$ correspondingly.

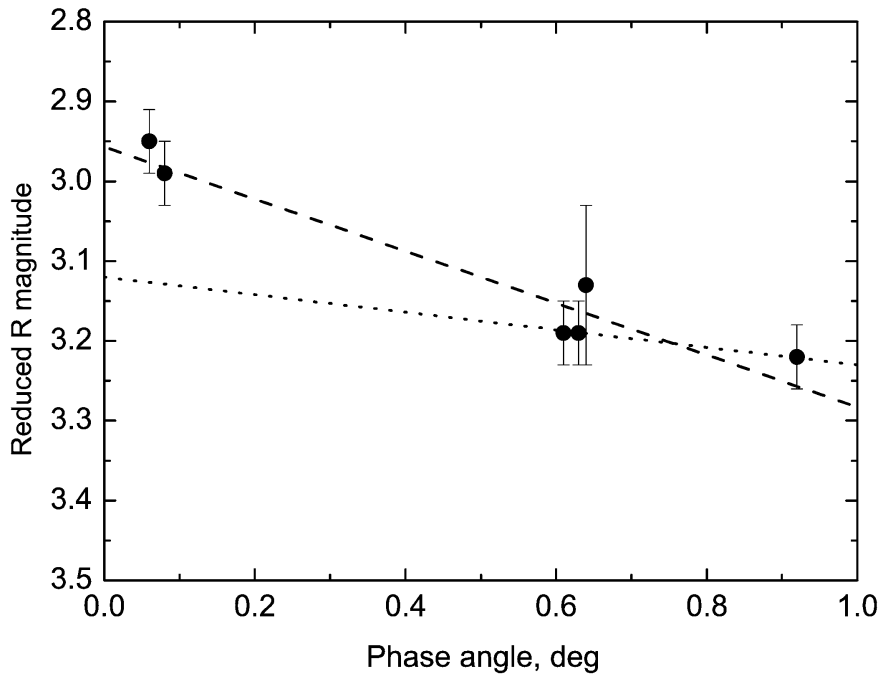

Fig. 2. Magnitude-phase dependence of Varuna in the maximum of lightcurve in the $\mathrm{R}$ band fitted by the linear fit to all data (dashed line) and to the data excluding phase angles less than $0.1 \mathrm{deg}$ (dotted line).

data obtained at Rozhen observatory (December 8) due to problems with their absolute magnitude calibration. For all other observations the uncertainty of correction for the lightcurve amplitude is less than the estimated uncertainty of our absolute photometry. The obtained magnitude-phase dependence is shown in Fig. 2. Magnitude increases in $0.24 \mathrm{mag}$ when phase angle decreases from 0.92 to $0.06 \mathrm{deg}$. The linear fit to all data yields a phase coefficient of $0.33 \pm 0.05 \mathrm{mag} / \mathrm{deg}$ while the same fit to the data excluding phase angles less than 0.1 deg results in smaller phase coefficient of $0.10 \pm 0.16 \mathrm{mag} / \mathrm{deg}$. The last case is preferable (Fig. 2), however more observational data are needed to separate between linear and non-linear behavior of the phase curve. As discussed in Section 4, the addition of data from other authors rules out a simple linear behavior and confirms an existence of an opposition surge. 


\section{Lightcurve changes toward zero phase angle}

\subsection{Lightcurves of 20000 Varuna}

Varuna, as one of the brightest known trans-neptunian objects, was intensively observed after its discovery in 2000. It belongs to "classical TNOs" dynamical group. Its size and albedo were measured repeatedly: a mean diameter of $900 \mathrm{~km}$ with an albedo of 0.07 in the $\mathrm{R}$ band was determined by Jewitt et al. (2001) and a mean diameter of $1060 \mathrm{~km}$ with an albedo of 0.038 ( $\mathrm{V}$ band) and 0.049 ( $\mathrm{R}$ band) was obtained by Lellouch et al. (2002). Recently it was announced that Varuna may have a higher visible albedo of $0.12-0.30$ and a smaller size of 450 $700 \mathrm{~km}$ than previously determined (Stansberry et al., 2005).

Farnham (2001) was the first who reported a fast rotation of Varuna with a single-peaked period of $3.17 \mathrm{~h}$ and $0.5 \mathrm{mag}$ amplitude. Jewitt and Sheppard (2002) observed the object during 7 nights in 2001 and determined a rotation period of $6.3442 \mathrm{~h}$ (double-peaked lightcurve) and amplitude of 0.42 mag. They also searched for rotational variations in colors (BVRI) but did not find any variations correlated with Varuna's rotation. Ortiz et al. (2003) made observations of Varuna in 2002 during two nights and found a rotation period of $6.3436 \mathrm{~h}$ after combining their data with previous observations by Jewitt and Sheppard (2002). Further photometric observations of Varuna, with an emphasis on extremely small phase angles, were made in 2002/2003 by Hicks et al. (2005). Thus, Varuna represents an unprecedented case among trans-neptunian objects being continuously observed for 5 years. Below we analyze the available set of Varuna's photometric data.

The available data allowed us to improve the accuracy of the rotation period determination. We found an unambiguous rotation period of $6.34358 \pm 0.00002 \mathrm{~h}$. All available observations agree well with this period value indicating a stable rotation state of Varuna. Observations were done practically at the same aspect angle, i.e., the angle between the line of sight and the rotation axis, which has not noticeably changed from 2001 to 2005 due to the remoteness of the object.

The available observations let us to construct full period Varuna's lightcurves at different phase angles. Individual lightcurves were fitted by the Fourier series similar to the procedure described by Harris et al. (1989a). The fitted curves for different phase angles are shown in Fig. 3. Two lightcurves measured at the phase angle of $1.0^{\circ}$ in the $\mathrm{R}$ filter (Jewitt and Sheppard, 2002) and at the phase angle of $0.8^{\circ}$ without any filter in the wavelength range of $0.35-0.94 \mu \mathrm{m}$ with the maximum sensitivity of $0.58 \mu \mathrm{m}$ (Ortiz et al., 2003) show a remarkable coincidence with each other. The composite lightcurve at very small phase angle of $0.1 \mathrm{deg}$ was obtained by combining our observations with data from Hicks et al. (2005). A difference between lightcurves at very small and at larger phase angles is clearly seen both in amplitude and in extrema position (Fig. 3). Surprisingly, the lightcurve amplitude increased toward small phase angles. It cannot be explained by a complex shape of Varuna. The amplitude of lightcurves caused by rotation of an elongated body with homogeneous surface increases for large phase angles, as observed for asteroids (e.g., Zappala

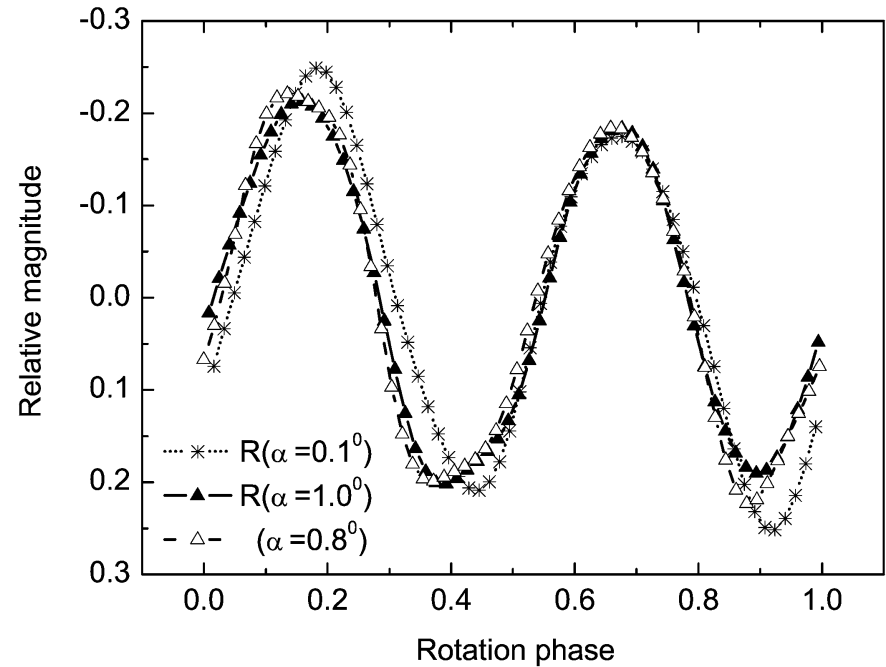

Fig. 3. Composite lightcurves of Varuna at different phase angles constructed by the Fourier fit to individual lightcurves.

et al., 1990). Moreover, the range of observed phase angles (0.1-1 deg) is so small that the only plausible explanation for lightcurve changes is the variation of light-scattering properties over the surface.

In fact, lightcurves observed very close to opposition are the least suitable for shape determination since the shape effects are more pronounced at large phase angles (Kaasalainen and Torppa, 2001). The lightcurve at opposition can give an indication on surface albedo variegation. For regolith-like surfaces the scattering is very close to nearly geometric at opposition, this means that the observed brightness is proportional to the product of the visible area and the visual surface albedo (Lupishko et al., 1983; Kaasalainen et al., 1992). Thus, any difference in magnitude between opposite sides of the body (half-period interval in the lightcurve) can be treated as indicative (i) on surface albedo variegation at the equatorial aspect of view, (ii) on non-convex shape and/or albedo variegation at other aspects, and (iii) on surface scattering very different from the geometric one.

For the particular case of Varuna its large lightcurve amplitude is in a favor of a nearly equatorial aspect of observations. Thus, an increase in asymmetry of Varuna's lightcurve toward zero phase angle is probably attributed to variations of the scattering properties over Varuna's surface. The observed difference in magnitude between opposite sides is rather small (about $0.07 \mathrm{mag}$ ) as compared to the overall lightcurve amplitude $(0.42 \mathrm{mag})$ and was detected due to numerous observations of Varuna at various phase angles. In the case of a low amplitude lightcurve similar variations in surface scattering properties could produce noticeable lightcurve changes toward zero phase angle. We have checked whether the changes observed in the lightcurve amplitude for TNO $1996 \mathrm{TO}_{66}$ can be caused by the same reason.

\subsection{Lightcurves of $193081996 \mathrm{TO}_{66}$}

The trans-neptunian object 193081996 TO $_{66}$ also belongs to the "classical TNOs" dynamical class. Assuming an albedo 
of 0.04 its estimated diameter is $652 \mathrm{~km}$ (Hainaut et al., 2000). The first lightcurve measurements for this object were made by Hainaut et al. (2000) during three observing runs in 1997-1998. They found a rotation period of $6.25 \pm 0.03 \mathrm{~h}$ and noticed a significant change of lightcurve shape with an amplitude varying from 0.12 to $0.33 \mathrm{mag}$. As a possible explanation they considered cometary activity of the object during the observations. Sekiguichi et al. (2002) verified the lightcurve changes of 1996 TO$_{66}$ in the 1999 observing run and found a consistency with the previously determined value of rotation period and a lightcurve amplitude of $0.21 \mathrm{mag}$. Search for cometary activity did not give positive result. In 2001 observations of 1996 TO$_{66}$ were made by Sheppard and Jewitt (2003). They determined a rotation period of $7.92 \pm 0.04 \mathrm{~h}$ and a 0.26 mag lightcurve amplitude. Sheppard and Jewitt (2003) also analyzed all available data and did not find any evidence for a change of rotation period while amplitude may have changed.

We reanalyzed available photometric data of $1996 \mathrm{TO}_{66} \mathrm{ob}-$ tained in 1997-1998 (Hainaut et al., 2000), in 1999 (Sekiguichi et al., 2002), and in 2001 (Sheppard and Jewitt, 2003). It occurs that the 1998 observations which showed the largest lightcurve amplitude of 0.33 mag were obtained very close to opposition: at phase angle of about $0.1^{\circ}$. The other observations were made at larger phase angles from $0.4^{\circ}$ to $1^{\circ}$. The large amplitude observed at $0.1^{\circ}$ of phase angle can be explained by opposition effect phenomenon in the case of considerable variations of the scattering properties over the surface. Different values of opposition surge for surfaces seen in lightcurve extrema will result in an increase of lightcurve amplitude compared to that at larger phase angles.

Using available data it is impossible to determine unambiguously a rotation period of $1996 \mathrm{TO}_{66}$. Sheppard and Jewitt (2003) determined a rotation period of $7.92 \mathrm{~h}$ but pointed out other plausible periods, namely 5.9, 7.92, and $9.6 \mathrm{~h}$. Combining of all available observations of $1996 \mathrm{TO}_{66}$ and taking into account phase angle effect we found one more plausible rotation period of about $11.9 \mathrm{~h}$. The composite lightcurve based on this period value is shown in Fig. 4. Note that the rotation period is close to $12 \mathrm{~h}$ and observations during the same observing run usually covered practically the same interval of rotation phases. In that case small lightcurve amplitude of $1996 \mathrm{TO}_{66}$ observed in 1997 can be explained by a short duration of observations covered a broad maximum of object's lightcurve. As one can see from Fig. 4, all observations agree well in lightcurve minima while the discrepancy in the primary maximum can be caused by phase angle effect. Further observations are needed to determine unambiguous value of rotation period of $1996 \mathrm{TO}_{66}$ and to confirm considerable variations of the scattering properties over its surface.

Lightcurve observations of TNOs at extremely low phase angles $\left(\leqslant 0.1^{\circ}\right)$ can undergo an influence of opposition effect phenomenon. If an increase in brightness due to the opposition effect varies over the object's surface it leads to an increase in the value of lightcurve amplitude measured at extremely low phase angles.

We have pointed out an effect of lightcurve changes at extremely low phase angles, which needs in verification. If it will

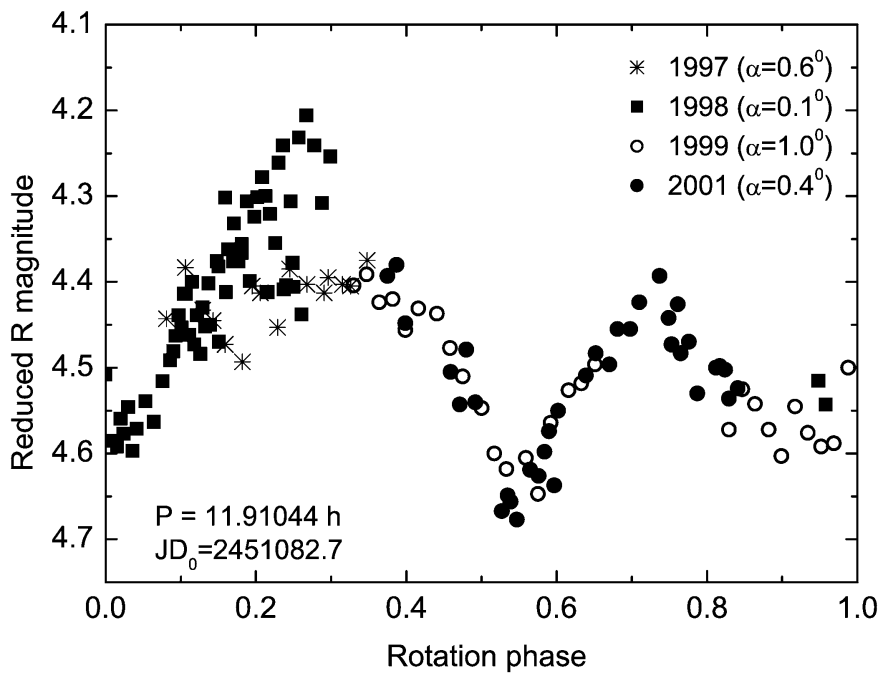

Fig. 4. Composite lightcurve of $193081996 \mathrm{TO}_{66}$ with the rotation period of 11.91044 h combined observations in 1997 and 1998 by Hainaut et al. (2000), in 1999 by Sekiguichi et al. (2002), and in 2001 by Sheppard and Jewitt (2003). The year and mean phase angle of observations are given in the upper corner of the figure.

be confirmed by further observations it gives an interesting approach to study variations of the surface scattering properties of trans-neptunian objects.

\section{Opposition effect}

The above-mentioned changes of TNO's lightcurve amplitude with phase angle make more complicated a study of their opposition effect behavior. A thorough correction of observations at different phase angles to correspond to the same side of the object is needed. In the Varuna's case there is enough data for such correction while for TNO $1996 \mathrm{TO}_{66}$ observations at different phase angles are related to different parts of the surface.

The magnitude-phase curves for opposite sides of Varuna are given in Fig. 5. They were calculated for the primary (M1) and secondary (M2) maxima of the lightcurve from both published and new observations of Varuna. We do not use observations in which a lightcurve maximum was not observed. The uncertainty of each point in Fig. 5 does not exceed 0.04 mag. One can see that observations by different authors are in a good agreement. They clearly show a non-linear increase in Varuna's magnitude at very low phase angles. There is a small but systematic difference between phase curves of surface hemispheres corresponding to the primary and to the secondary maxima of lightcurve. The phase curves were fitted by a combination of linear and exponential functions similar to that proposed by Kaasalainen et al. (2003). The difference is that we applied it directly to magnitudes (not transformed to intensities) and used the fixed slope parameter (phase coefficient) defined from the linear fit of the data at phase angles larger $0.5 \mathrm{deg}$. Such procedure gave rather reasonable fitting of observational data as one can see from Fig. 5. The phase coefficient is the same for both maxima and it is equal to $0.11 \pm 0.03 \mathrm{mag} / \mathrm{deg}$. Opposition effect amplitude, defined as an excess in magnitude at zero 


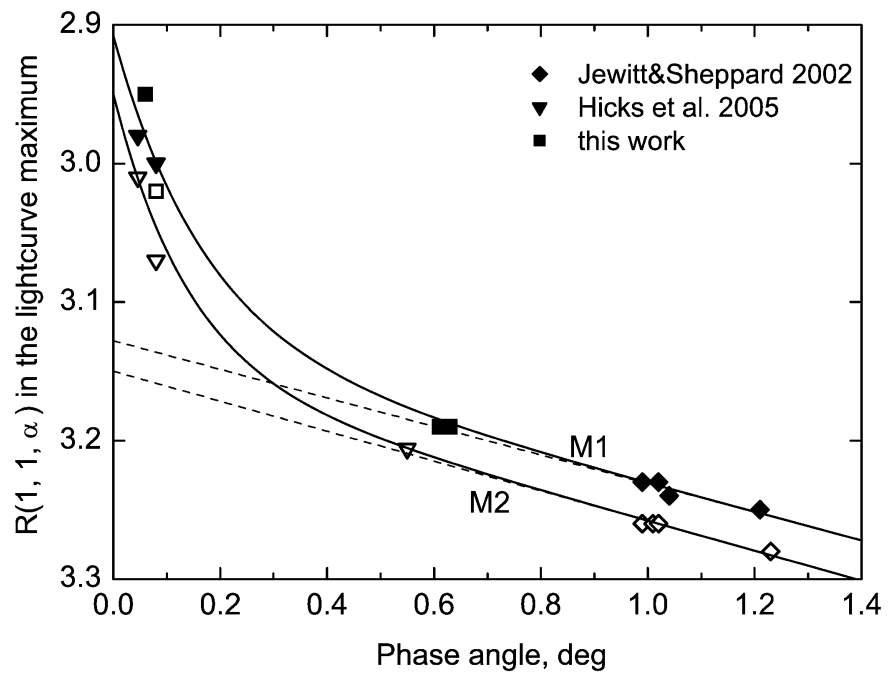

Fig. 5. Opposition effect of Varuna at the different lightcurve maxima fitted by linear-exponential fit.

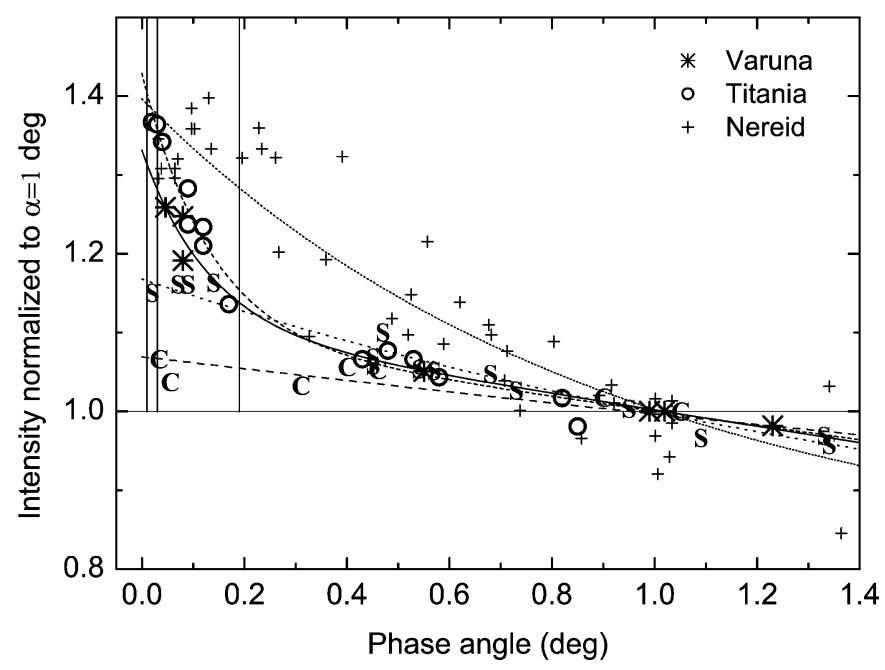

Fig. 6. Comparison of opposition effects of Varuna, selected asteroids of $\mathrm{C}$ and $\mathrm{S}$ compositional types, uranian satellite Titania and neptunian satellite Nereid (for references see text). Linear-exponential fits correspond to C (dash line) and $\mathrm{S}$ (dot line) asteroids, Varuna (solid line), Titania (short dash line), and Nereid (short dot line). The vertical lines show the angular size of the solar disk at distances where Varuna, Titania, and asteroids were located during observations.

phase angle relatively to the extrapolation of the linear part of the phase curve, can reach $0.2 \pm 0.05 \mathrm{mag}$. There is a tendency for larger opposition effect amplitude for the surface seen in the primary lightcurve maximum. The width of opposition surge is uncertain due to the lack of observations at $0.2^{\circ}-0.5^{\circ}$ phase angles. Further observations are needed to determine the width, which is certainly less than $0.5^{\circ}$.

Varuna is the first trans-neptunian object for which an existence of a pronounced opposition surge in magnitude-phase dependence was proved independently by different observers (see also Hicks et al., 2005). Its amplitude reaches $0.2 \mathrm{mag}$ at zero phase angle relatively to the extrapolation of the linear part of the phase curve and its width is less than $0.5^{\circ}$. Such a narrow opposition surge was observed for bright asteroids and satellites of major planets (Harris et al., 1989b;
Buratti et al., 1992; Helfenstein et al., 1998; Verbiscer et al., 2005). It is usually explained by coherent backscatter enhancement which arises due to constructive interference of rays traveling in opposite paths through the medium (Muinonen, 1990; Shkuratov, 1991; Mishchenko and Dlugach, 1993; Hapke et al., 1998). Coherent backscattering and well-known shadowing effect are considered as main mechanisms of an opposition effect phenomena (e.g., Shkuratov et al., 1999; Hapke, 2002). It is widely assumed that coherent backscattering is responsible for a sharp and narrow intensity peak while shadowing effect results in a much wider peak (e.g., Helfenstein et al., 1997). A value of opposition effect from regolith-like surfaces varies with surface albedo and reaches the maximum value for moderate albedo surfaces (Belskaya and Shevchenko, 2000; Nelson et al., 2004).

To better understand characteristics of Varuna's opposition effect we have compared it to that of other Solar System bodies which were selected using the following criteria: (i) having surface albedo within the range assumed for Varuna, (ii) being representative of opposition effect amplitudes of Solar System bodies, (iii) having well-measured phase curve down to phase angles $<0.05^{\circ}$. Fig. 6 shows an intensity of scattered light normalized to that at $1^{\circ}$ of phase angle for selected asteroids belonging to $C$ type (albedo range is $0.04-0.07$ ) and $S$ type (albedo is 0.17-0.26). The data were taken from Dovgopol et al. (1992), Shevchenko et al. (2002, 2005), Belskaya et al. (2003b). We also plotted observations of two satellites of major planets which are known to have the largest amplitude of opposition surge ever observed for Solar System bodies (see also Schaefer and Rabinowitz, 2002). The data of the uranian satellite Titania having albedo of 0.27 were plotted according to Buratti et al. (1992). Observations of the neptunian satellite Nereid having albedo of 0.16 were taken from Schaefer and Tourtellotte (2001). All data are well fitted by a linearexponential function proposed by Kaasalainen et al. (2003). Note that Nereid's atypical phase curve behavior needs in verification since available observations have significant scatter (see also Grav et al., 2003). All other objects show rather similar phase behavior down to phase angles of 0.3-0.4 deg while at smaller phase angles their phase curves become noticeably different.

To compare the opposition effect behavior at very small phase angles we need to take into account a finite angular size of the solar disk. Non-zero angular size of the light source results in smoothing of opposition peak due to integration of phase angles over the solar disk (e.g., Shkuratov and Stankevich, 1995). The vertical lines in Fig. 6 show the angular size of the solar disk $\alpha_{0}$ at distances where Varuna, Titania and asteroids (the mean distance for the observed objects) were located during observations. The smoothing of an opposition peak is well seen for the S-type asteroids whose phase curves revealed practically the same level of intensity at $\alpha<\alpha_{0}$. An estimation of the effect following by Shkuratov and Stankevich (1995) showed that it is important only at phase angles less than the angular radius of the Sun (see also Helfenstein et al., 1997). Thus, the effect of a finite angular size of the solar disk for asteroids can be neglected down to the phase angle of $0.1^{\circ}$. 
One can see that the trans-neptunian object is characterized by an intermediate value of opposition peak showing stronger effect as compared to moderate albedo asteroids and smaller one in comparison to satellites. Opposition effect observed for Varuna is essentially different from that of dark asteroids which have less pronounced effect. It may be interpreted in favor of moderate surface albedo of Varuna. Such explanation is more plausible than an assumption of very fluffy Varuna's regolith in case of its low albedo (Belskaya et al., 2003a; Hicks et al., 2005). Recent measurements of Varuna's albedo with Spitzer telescope cast doubts on previously determined low values of its albedo equal to 0.04-0.07 (Jewitt et al., 2001; Lellouch et al., 2002) and give an albedo of 0.14 as the most probable value but with rather large uncertainty (D.P. Cruikshank, private communication). The large amplitude of Varuna's opposition effect is quite typical for moderate albedo Solar System bodies.

Recent observations of other trans-neptunian object 55637 (2002 $\mathrm{UX}_{25}$ ) also indicated a possibility of an opposition surge at phase angles less than $0.1^{\circ}$ (Rousselot et al., 2005). Further observations of TNOs are needed to estimate values of opposition effect and their correlations with surface albedo.

Another important question connected with the opposition effect observations is the determination of absolute magnitudes of TNOs. They can be about 0.2 mag brighter as compared to values based on the linear fit of magnitude-phase dependence.

\section{Conclusions}

Analysis of both new and available photometric observations of Varuna has shown an importance of low phase angle effects in photometry of trans-neptunian objects.

1. A large opposition surge was found which can reach 0.2 mag relatively to the extrapolation of the linear part of the phase curve to zero phase angle. It width is less than $0.5 \mathrm{deg}$. The opposition effect of Varuna is markedly different from that of dark asteroids while it is quite typical for moderate albedo Solar System bodies. It may be considered in favor of moderate surface albedo of Varuna. Such explanation is more plausible than an assumption of particular structure of Varuna's surface in case of its low albedo.

2. An indication on variations of the scattering properties over Varuna's surface was noticed, which resulted in an increase of the lightcurve amplitude toward zero phase angle. In the case of a low amplitude lightcurve similar variations of surface scattering properties can produce noticeable lightcurve changes at phase angles close to zero. It was shown that the a phase effect similar to that of Varuna can be responsible for lightcurve changes found for TNO 19308 (1996 TO 66 ) in 1997-1999. Observations at the phase angles of $0.1 \mathrm{deg}$ and less should be taken with a great care for composite lightcurve construction.

Further observations of trans-neptunian objects at very low phase angles are needed to understand whether a large opposi- tion surge and variegation of surface scattering properties are common events among TNOs.

\section{Acknowledgments}

This research was partially based on data obtained at the Observatorio de Sierra Nevada, which is operated by the Instituto de Astrofísica de Andalucía, CSIC. We are very grateful to Michael Hicks and Scott Sheppard for providing us with original observational data and to Yurij Krugly for valuable comments. Ukrainian team was partly supported by Ukrainian foundation of fundamental research (Grant No. 02.07/379). J.L.O. acknowledges support from AYA-2002-00382, AYA2005-07808-C03-01, and FEDER funds. N.P. acknowledges funding from European Social Fund and FCT, Portugal, ref: BPD/18729/2004.

\section{References}

Barucci, M.A., Doressoundiram, A., Cruikshank, D.P., 2004. Surface characteristics of transneptunian objects and centaurs from photometry and spectrophotometry. In: Festou, M. (Ed.), Comets II. Univ. of Arizona Press, Tucson, pp. 647-658.

Belskaya, I.N., Shevchenko, V.G., 2000. Opposition effect of asteroids. Icarus 147, 94-105.

Belskaya, I.N., Barucci, M.A., Shkuratov, Yu.G., 2003a. Opposition effect of Kuiper belt objects: Preliminary estimations. Earth Moon Planets 92, 201206.

Belskaya, I.N., Shevchenko, V.G., Kiselev, N.N., Krugly, Yu.N, Shakhovskoy, N.M., Efimov, Yu.S., Gaftonyuk, N.M., Cellino, A., Gil-Hutton, R., 2003 b. Opposition polarimetry and photometry of S- and E-type asteroids. Icarus $166,276-284$.

Buratti, B.J., Gibson, J., Mosher, J.A., 1992. CCD photometry of the uranian satellites. Astron. J. 104, 1618-1622.

Dovgopol, A.N., Krugly, Yu.N., Shevchenko, V.G., 1992. Asteroid 126 Velleda: Rotation period and magnitude-phase curve. Acta Astron. 42, 67-72.

Farnham, T.L., 2001. (20000) $2000 \mathrm{WR}_{106}$. IAU Circ. 7583, 4.

Grav, T., Holman, M.J., Kavelaars, J.J., 2003. The short rotation period of Nereid. Astrophys. J. 591, L71-L74.

Jewitt, D.C., Sheppard, S.S., 2002. Physical properties of trans-neptunian object (20000) Varuna. Astron. J. 123, 2110-2120.

Jewitt, D., Aussel, H., Evans, A., 2001. The size and albedo of the Kuiper-belt object (20000) Varuna. Nature 411, 446-447.

Hainaut, O.R., Delahodde, C.E., Boehnhardt, H., Dotto, E., Barucci, M.A., Meech, K.J., Bauer, J.M., West, R.M., Doressoundiram, A., 2000. Physical properties of TNO 1996 TO $_{66}$. Lightcurves and possible cometary activity. Astron. Astrophys. 356, 1076-1088.

Hapke, B., 2002. Bidirectional reflectance spectroscopy. 5. The coherent backscatter opposition effect and anisotropic scattering. Icarus 157, 523534

Hapke, B., Nelson, R., Smythe, W., 1998. The opposition effect of the Moon: Coherent backscatter and shadow hiding. Icarus 133, 89-97.

Harris, A.W., Young, J.W., Bowell, E., Martin, L.J., Millis, R.L., Poutanen, M., Scaltriti, F., Zappala, V., Schober, H.J., Debehogne, H., Zeigler, K.W., 1989a. Photoelectric observations of asteroids 3, 24, 60, 261, and 863. Icarus 77, 171-186.

Harris, A.W., Young, J.W., Contreiras, L., Dockweiler, T., Belkora, L., Salo, H., Harris, W.D., Bowell, E., Poutanen, M., Binzel, R.P., Tholen, D.J., Wang, S., 1989b. Phase relations of high albedo asteroids: The unusual opposition brightening of 44 Nysa and 64 Angelina. Icarus 81, 365-374.

Helfenstein, P., Veverka, J., Hillier, J., 1997. The lunar opposition effect: A test of alternative models. Icarus 128, 2-14.

Helfenstein, P., Currier, N., Clark, B.E., Veverka, J., Bell, M., Sullivan, R., Klemaszewski, J., Pappalardo, R.R.T., Head, J.W., Jones, T., Klaasen, K., Magee, K., Geissler, P., Greenberg, R., McEwen, A., Phillips, C., Colvin, 
T., Davies, M., Denk, T., Neukum, G., Belton, M.J.S., 1998. Galileo observations of Europa's opposition effect. Icarus 135, 41-63.

Hicks, M.D., Simonelli, D.P., Buratti, B.J., 2005. Photometric behavior of 20000 Varuna at very small phase angles. Icarus 176, 492-498.

Kaasalainen, M., Torppa, J., 2001. Optimization methods for asteroid lightcurve inversion. I. Shape determination. Icarus 153, 24-36.

Kaasalainen, M., Lamberg, L., Lumme, K., Bowell, E., 1992. Interpretation of lightcurves of atmosphereless bodies. I. General theory and new inversion schemes. Astron. Astrophys. 259, 318-332.

Kaasalainen, S., Piironen, J., Kaasalainen, M., Harris, A.W., Muinonen, K., Cellino, A., 2003. Asteroid photometric and polarimetric phase curves: Empirical interpretation. Icarus 161, 24-46.

Krugly, Yu.N., 2003. Photometry of near-Earth asteroids. Ph.D. thesis, Astronomical Institute of Kharkiv National University, Kharkiv.

Landolt, A.U., 1992. UBVRI photometric standard stars in the magnitude range 11.5-16.0 around the celestial equator. Astron. J. 104, 436-491.

Lellouch, E., Moreno, R., Ortiz, J.L., Paubert, G., Doressoundiram, A., Peixinho, N., 2002. Coordinated thermal and optical observations of transneptunian object (20000) Varuna from Sierra Nevada. Astron. Astrophys. 391, 1133-1139.

Lupishko, D.F., Akimov, L.A., Belskaya, I.N., 1983. On photometric heterogeneity of asteroid surfaces. In: Proceedings ACM 1983. Uppsala University, pp. 63-70.

Luu, J.X., Jewitt, D.C., 2002. Kuiper belt objects: Relics from the accretion disk of the Sun. Astron. Astrophys. Annu. Rev. 40, 63-101.

Mishchenko, M.I., Dlugach, J.M., 1993. Coherent backscatter and the opposition effect for E-type asteroids. Planet. Space Sci. 41, 173-181.

Muinonen, K., 1990. Light scattering by inhomogeneous media: Backward enhancement and reversal of polarization. Ph.D. thesis, University of Helsinki.

Nelson, R.M., Hapke, B.W., Smythe, W.D., Hale, A.S., Piatek, J.L., 2004. Planetary regolith microstructure: An unexpected opposition effect result. Lunar Planet. Sci. 35. Abstract N1089.

Ortiz, J.L., Gutiérrez, P.J., Casanova, V., Sota, A., 2003. A study of short term rotational variability in TNOs and Centaurs from Sierra Nevada Observatory. Astron. Astrophys. 407, 1149-1155.

Rousselot, P., Petit, J.-M., Poulet, F., Lacerda, P., Ortiz, J., 2003. Photometry of the Kuiper-belt object $1999 \mathrm{TD}_{10}$ at different phase angles. Astron. Astrophys. $407,1139-1147$.
Rousselot, P., Petit, J.-M., Poulet, F., Sergeev, A., 2005. Photometric study of Centaur (60558) $2000 \mathrm{EC}_{98}$ and trans-neptunian object (55637) $2002 \mathrm{UX}_{25}$ at different phase angles. Icarus 176, 478-491.

Schaefer, B.E., Rabinowitz, D.L., 2002. Photometric light curve for the Kuiper belt object $2000 \mathrm{~EB}_{173}$ on 78 nights. Icarus 160, 52-58.

Schaefer, B.E., Tourtellotte, S.W., 2001. Photometric light curve for Nereid in 1998: A prominent opposition surge. Icarus 151, 112-117.

Sekiguichi, T., Boehnhardt, H., Hainaut, O.R., Delahodde, C.E., 2002. Bicolor lightcurve of TNO 1996 TO$_{66}$ with the ESO-VLT. Astron. Astrophys. 385, 281-288.

Sheppard, S.S., Jewitt, D.C., 2002. Time-resolved photometry of Kuiper belt objects: Rotations, shapes, and phase functions. Astron. J. 124, 1757-1775.

Sheppard, S.S., Jewitt, D.C., 2003. Hawaii Kuiper belt variability project: An update. Earth Moon Planets 92, 207-219.

Shevchenko, V.G., Belskaya, I.N., Krugly, Yu.N., Chiorny, V.G., Gaftonyuk, N.M., 2002. Asteroid observations at low phase angles. II. 5 Astraea, 75 Eurydike, 77 Frigga, 105 Artemis, 119 Althaea, 124 Alkeste and 201 Penelope. Icarus 153, 310-320.

Shevchenko, V.G., Chiorny, V.G., Gaftonyuk, N.M., Krugly, Yu.N., Belskaya, I.N., Tereschenko, I.A., Velichko, F.P., 2005. Opposition effect of dark asteroids. In: IAU Symposium 229: Asteroids, Comets, Meteors, August 7-12, 2005, Búzios, Rio de Janeiro, Brazil, p. 133 (abstract).

Shkuratov, Yu.G., 1991. An interference model of the negative polarization of light scattered by atmosphereless celestial bodies. Solar Syst. Res. 25, 134 142.

Shkuratov, Yu.G., Stankevich, D.G., 1995. Can lunar opposition spike measured by Clementine exist? Lunar Planet. Sci. 26. Abstract 1295.

Shkuratov, Yu.G., Kreslavsky, M.A., Ovcharenko, A.A., Stankevich, D.G., Zubko, E.S., Pieters, C., Arnold, G., 1999. Opposition effect from Clementine data and mechanisms of backscatter. Icarus 141, 132-155.

Stansberry, J.A., Cruikshank, D.P., Grundy, W.G., Margot, J.L., Emery, J.P., Fernandez, Y.R., Rieke, G.H., 2005. Albedos, diameters (and a density) of Kuiper belt and Centaur objects. Bull. Am. Astron. Soc. 37. Abstract 52.05.

Verbiscer, A.J., French, R.G., McGhee, C.A., 2005. The opposition surge of Enceladus: HST observations 338-1022 nm. Icarus 173, 66-83.

Zappala, V., Cellino, A., Barucci, M.A., Fulchignoni, M., Lupishko, D.F., 1990. An analysis of the amplitude-phase relationship among asteroids. Astron. Astrophys. 231, 548-560. 